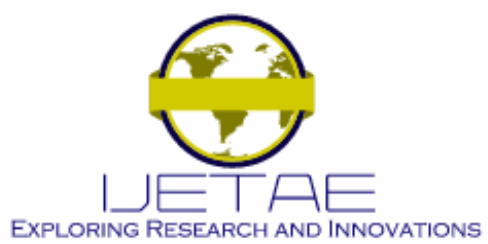

International Journal of Emerging Technology and Advanced Engineering

Website: www.ijetae.com (E-ISSN 2250-2459, Scopus Indexed, ISO 9001:2008 Certified Journal, Volume 11, Issue 08, August 2021)

Manuscript Received: 05 July 2021, Received in Revised form: 07 August 2021, Accepted: 12 August 2021

DOI: 10.46338/ijetae0821_01

\title{
Dynamic Model of TRMS Based on Homogeneous Transformation and Euler-Lagrange Equation
}

\author{
Nguyen Thi Le $\mathrm{Na}^{1}$, Vu Quoc Huy ${ }^{2}$ \\ ${ }^{1,2}$ Control, Automation in Production and Improvement of Technology Institute (CAPITI), No. 89B Ly Nam De, Hanoi, Vietnam \\ ${ }^{1}$ lenacapitegmail.com; ${ }^{2}$ maihuyvulgmail.com
}

\begin{abstract}
The aerodynamic experimental set TRMS (Twin Rotor MIMO System) is a strong nonlinear system, which has been taken by many scientists as an object to test modern control algorithms. The paper built a complete and explicit dynamic model for the TRMS based on dissociating TRMS into 3 subsystems, using homogeneous transformations in orthogonal coordinate systems to calculate the mass point's position and velocity in component sub-systems; Euler Lagrange equation was applied to model the dynamics for the object.
\end{abstract}

Keywords-TRMS, dynamics, homogeneous transformation, mechanical system dissociation, Euler Lagrange.

\section{INTRODUCTION}

Feedback Instrument Ltd's Twin Rotor MIMO System (TRMS) is an open-structured experimental set that has installed PID control algorithms, visually describing a twin-rotor helicopter flying instrument [1], [2]. This is a strong nonlinear object with interleaving, so although it was designed and manufactured in 1998, so far TRMS has been used by many researchers as a control object for testing control algorithms. At the forefront of TRMS research is the manufacturer Feedback Instrument Ltd. The company built a mathematical model for TRMS based on Newtonian mechanics, but that mathematical model did not consider the length of the rotating pin and ignores the inertia moment of some small components in the system. To develop and test some algorithms for linear control (PID, LQR, and LQG), nonlinear control (sliding mode, adaptive control, fuzzy, neuron, feedback linearization, etc.), many scholars have researched, built, and adjusted the mathematical model of TRMS under specific conditions. Due to the use of some assumptions when building the model, the structure of the model would be incomplete. Therefore, to develop a control algorithm for TRMS with better output response quality, it is necessary to have an accurate and suitable model with few assumptions.
In this paper, we propose a new approach, whereby we decomposed the system of TRMS into subsystems, then we built a mathematical model of TRMS based on a homogeneous coordinate transformation and the EulerLagrange equation. The decomposition of TRMS into components helped the modeling process not to miss a single mechanical detail of the system, thereby build a full dynamics model for TRMS with control torque input, creating a basis for the development and testing of advanced direct torque control algorithms.

The paper was organized as follows: After the introduction, we conducted some literature review in the second section. The third section summarized the physical structure of TRMS. The dynamic model of the TRMS mechanical system was presented in the fourth section and the last section ended with some conclusions.

\section{LITERATURE REVIEW}

To design suitable controllers for the object, a mathematical model of the object must be obtained. There were two types of modeling conducted for TRMS: exact modeling and approximate modeling. To accurately model the nonlinear kinematics of TRMS, researchers used precise modeling based on Euler - Newton or Euler Lagrange method. Both of these methods gave accurate object description results and the difference between the two models was very small. In [3] and [4], the authors conducted the modeling according to both Euler - Newton, and Euler - Lagrange methods. The modeling based on the Euler - Lagrange method gave slightly more accurate results than Newton - Euler when compared to the real TRMS model. Research [5] built a white box model for TRMS based on the $2^{\text {nd }}$ Newton law and quantified the model using the recognition tool in MATLAB to produce a gray-box model for TRMS with a voltage control input - a gray-box regression model similar to the study [6] and [7]. When choosing a regression model, to some extent, the studies [5], [6], and [7] lost the completeness of the mathematical model. 


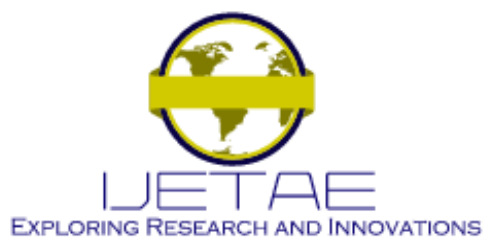

\section{International Journal of Emerging Technology and Advanced Engineering}

Website: www.ijetae.com (E-ISSN 2250-2459, Scopus Indexed, ISO 9001:2008 Certified Journal, Volume 11, Issue 08, August 2021)

Research [8] built a complete dynamic model based on the Euler - Lagrange equation and normalize the model by PD control action. However, because the control signal of the TRMS was voltage, the system had to go through voltage-torque conversion and this stage was not been considered in the modeling process.

In this paper, we used the modeling results based on the Euler - Lagrange method presented in [3] and [4] to build a mathematical model based on the Lagrange equation with the form of control moment inputs. We divided TRMS into 3 parts: (i) The first part included the beam and the other mechanism attached to it (main rotor and tail rotor, main fender, tail fender); (ii) The second part consisted of a counterweight and counterweight bar; and (iii) The last part was the pivot pin.

\section{PHYSICAL STRUCTURE OF TRMS}

The physical structure of the TRMS system was shown in Figure 1.

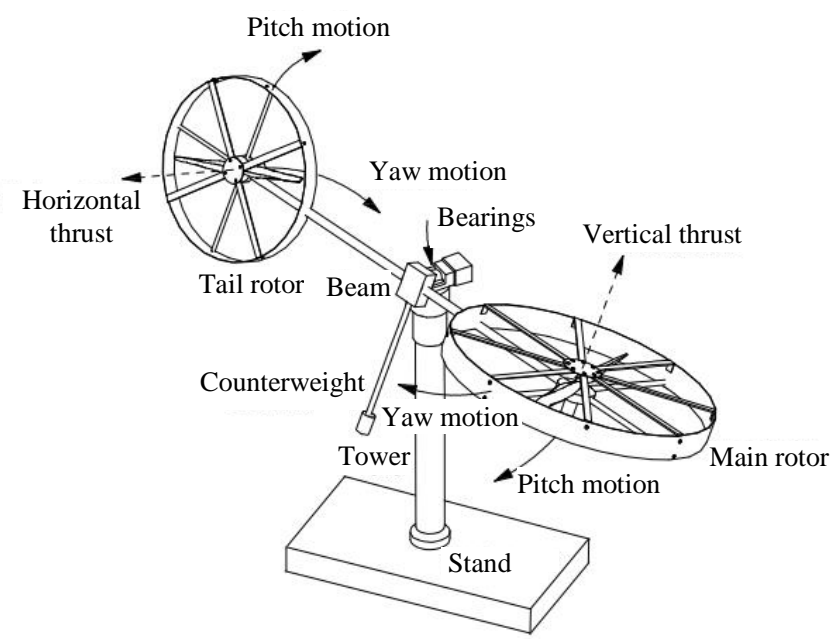

Figure 1. The Physical Structure of TRMS [9]

TRMS has the main rotor to change the pitch angle $\varepsilon$ and a tail rotor to change the yaw angle $\beta$. These two propellers attach to the beam and similar to the helicopter, they are perpendicular to each other. Each rotor is independently driven by a permanently excited DC motor, which can change the direction of rotation and speed. Each motor is attached to a tachometer to measure the angular rate of the propeller. The counterweight bar is attached to the beam at the position of the bearing.
The counterweight position on the counterweight bar can be changed to serve the experiment to change the kinematic parameters of the object. The entire structure is attached to a tower and can rotate around the tower in the horizontal plane. In addition, the system has optical encoders to measure the elevation angle $\varepsilon$ and azimuth angle $\beta$.

\section{DYNAMIC MODEL OF TRMS MECHANICAL SYSTEM}

\section{A. Theoretical basis}

The Euler-Lagrange equation of a mechanical system has the following expression:

$$
\begin{aligned}
& \underline{\boldsymbol{\tau}}=\frac{d}{d t}\left(\frac{\partial L}{\partial \underline{\dot{\boldsymbol{q}}}}\right)^{T}-\left(\frac{\partial L}{\partial \underline{\boldsymbol{q}}}\right)^{T} \\
& L=K-V \\
& \left\{\begin{array}{l}
K=\frac{1}{2} \int v^{2}(P) d m(P) \\
V=g \int r_{z}(P) d m(P)
\end{array}\right.
\end{aligned}
$$

Where:

$\boldsymbol{q}=\left[q_{1}, q_{2}, \ldots, q_{n}\right]^{T}$ is a joint variable vector;

$\underline{\boldsymbol{\tau}}=\left[\tau_{1}, \tau_{2}, \ldots, \tau_{n}\right]^{T}$ is an input torque vector;

$L$ is the Lagrange function;

$K$ is the kinetic the mechanical system;

$V$ is the potential energy of the mechanical system;

$P$ is a mass point in the system.

Suppose, in space, from the coordinate system attached to the body Bxyz, it was moved to the standard coordinate system $O X Y Z$ by 2 rotations and one translation in the order: Rotation $R_{z}(\beta)$ around the $O Z$ axis at an angle $\beta$, the rotation $R_{x}(\varepsilon)$ around the $O X$ axis at an angle $\varepsilon$ and the translation $T_{x y z}\left(x_{0}, y_{0}, z_{0}\right)$ along the axes of $O X Y Z$ with coordinates $\left(x_{0}, y_{0}, z_{0}\right)$ then in a homogeneous coordinate system we had the transformation $T$ as (4):

$$
T=R_{z}(\beta) R_{x}(\varepsilon) T_{x y z}\left(x_{0}, y_{0}, z_{0}\right)
$$

Where:

Rotation $R_{z}(\beta)$ around the $O Z$ axis: 


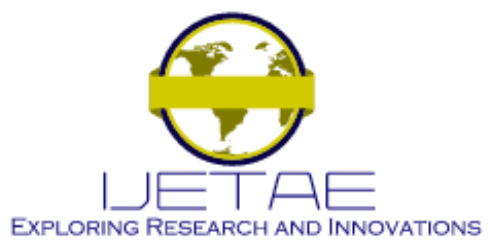

International Journal of Emerging Technology and Advanced Engineering

Website: www.ijetae.com (E-ISSN 2250-2459, Scopus Indexed, ISO 9001:2008 Certified Journal, Volume 11, Issue 08, August 2021)

$$
R_{z}(\beta)=\left[\begin{array}{cccc}
\cos \beta & \sin \beta & 0 & 0 \\
-\sin \beta & \cos \beta & 0 & 0 \\
0 & 0 & 1 & 0 \\
0 & 0 & 0 & 1
\end{array}\right]
$$

Rotation $R_{x}(\varepsilon)$ around the $O X$ axis:

$$
\begin{aligned}
& R_{x}(\varepsilon)=\left[\begin{array}{cccc}
1 & 0 & 0 & 0 \\
0 & \cos \varepsilon & \sin \varepsilon & 0 \\
0 & -\sin \varepsilon & \cos \varepsilon & 0 \\
0 & 0 & 0 & 1
\end{array}\right] \\
& T_{x y z}\left(x_{0}, y_{0}, z_{0}\right)=\left[\begin{array}{cccc}
1 & 0 & 0 & x_{0} \\
0 & 1 & 0 & y_{0} \\
0 & 0 & 1 & z_{0} \\
0 & 0 & 0 & 1
\end{array}\right]
\end{aligned}
$$

\section{B. Mathematical Model of TRMS}

In Figure 2, we define the orthogonal coordinate systems as follows: The standard coordinate system $O X Y Z$ has the center $O$ coincides with the yaw motion of the whole system, the $X O Y$ plane is the horizontal plane, the $O X$ axis is specified at $0^{\circ}$ of azimuth angle. The $B x y z$ coordinate system attached to the TRMS has the $B x$ axis in the direction of the extension of the pivot rod, the $B y$ axis along the beam towards the right, and the $B z$ axis upward in the direction of the extension of the counterweight bar. Thus, if $O X Y Z$ is rotated around the $O Z$ axis by an angle $\beta$, then $O X$ coincides with $B x$; next rotates around the $O X$ axis by an angle $\varepsilon$, then $O X Y Z$ will be parallel to $B x y z$ and a distance from this system $O B=h$. If $O X Y Z$ is translated by a distance $h$ in the $O X$ direction, the $O X Y Z$ and Bxyz coordinate systems will coincide.

\section{Define Orthogonal Coordinate Systems}

Orthogonal coordinate systems associated with TRMS were performed in Fig. 2.

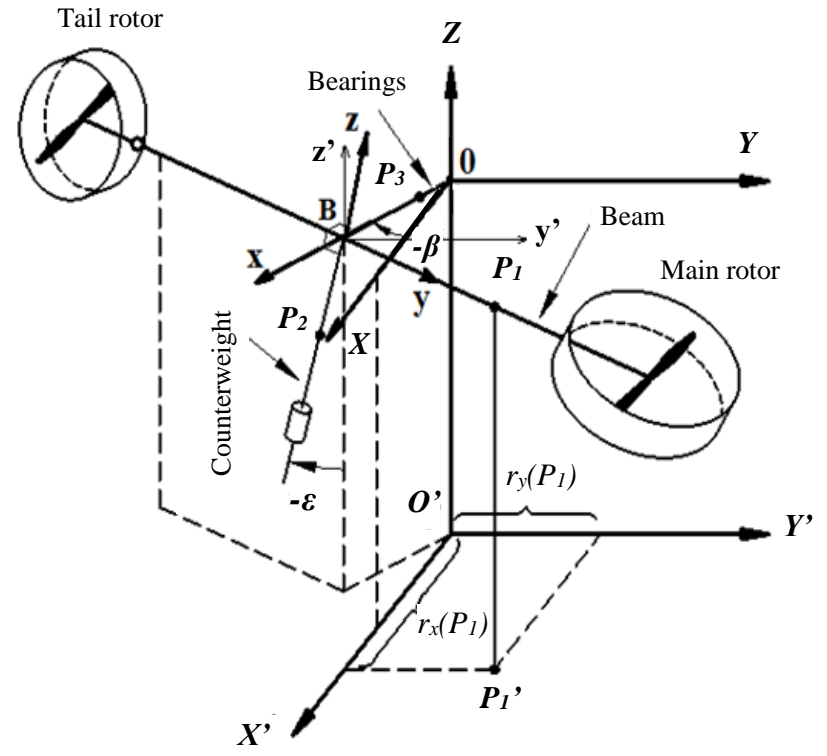

Figure 2. Orthogonal Coordinate Systems Associated with TRMS [4]

From Fig. 2, with the coordinate transformations (4) and (5a), (5b), (5c), knowing the angles $\beta, \varepsilon$ and then we could calculate the coordinates of any point $P$ on the TRMS according to (6).

$$
\left[\begin{array}{l}
X \\
Y \\
Z \\
1
\end{array}\right]=T\left[\begin{array}{l}
x \\
y \\
z \\
1
\end{array}\right]
$$

$$
T=\left[\begin{array}{cccc}
\cos \beta & \sin \beta \cos \varepsilon & \sin \beta \sin \varepsilon & h \cos \beta \\
-\sin \beta & \cos \beta \cos \varepsilon & \cos \beta \sin \varepsilon & -h \sin \beta \\
0 & -\sin \varepsilon & \cos \varepsilon & 0 \\
0 & 0 & 0 & 1
\end{array}\right]
$$

In this paper, we chose the approach of building a mathematical model of the TRMS system based on the Euler-Lagrange equation on the view of separating the TRMS system into 3 subsystems: 


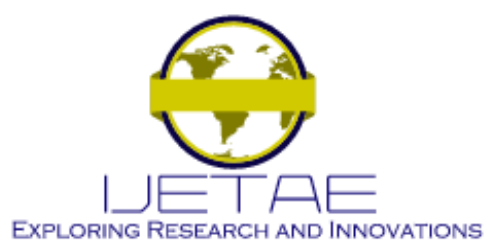

\section{International Journal of Emerging Technology and Advanced Engineering}

Website: www.ijetae.com (E-ISSN 2250-2459, Scopus Indexed, ISO 9001:2008 Certified Journal, Volume 11, Issue 08, August 2021)

The first one included the beam and the other mechanism attached to it (main rotor and tail rotor, main fender, tail fender); the second one included the counterweight bar and counterweight; the third one was the pivot pin.

\section{Position and Velocity of Subsystems in OXYZ}

To calculate the kinetic energy according to formula (2), we need to calculate the velocity of any mass point (every particle) on the TRMS.

a) Position and velocity in the $1^{\text {st }}$ subsystem:

In Figure 2, $P_{1}$ was a point on the horizontal bar located in the segment $B P_{I}=R_{1}$ from the center of $B$ whose coordinates in the Bxyz system were $\left[0, R_{l}, 0,1\right]^{\mathrm{T}}$. For convenience, we denoted the coordinates of $P_{1}$ in the $O X Y Z$ system as $\left[r_{x}\left(P_{l}\right), r_{y}\left(P_{l}\right), r_{z}\left(P_{l}\right)\right]$ and its velocity was respectively $\left[v_{x}\left(P_{I}\right), v_{y}\left(P_{I}\right), v_{z}\left(P_{I}\right)\right]$. From (6) we got:

$$
\begin{aligned}
{\left[\begin{array}{c}
r_{x}\left(P_{1}\right) \\
r_{y}\left(P_{1}\right) \\
r_{z}\left(P_{1}\right) \\
1
\end{array}\right] } & {\left[\begin{array}{cccc}
\cos \beta & \sin \beta \cos \varepsilon & \sin \beta \sin \varepsilon & h \cos \beta \\
-\sin \beta & \cos \beta \cos \varepsilon & \cos \beta \sin \varepsilon & -h \sin \beta \\
0 & -\sin \varepsilon & \cos \varepsilon & 0 \\
0 & 0 & 0 & 1
\end{array}\right]\left[\begin{array}{c}
0 \\
R_{1} \\
0 \\
1
\end{array}\right]_{(7)} } \\
& =\left[\begin{array}{c}
R_{1} \sin \beta \cos \varepsilon+h \cos \beta \\
R_{1} \cos \beta \cos \varepsilon-h \sin \beta \\
-R_{1} \sin \varepsilon \\
1
\end{array}\right]
\end{aligned}
$$

Take the position derivative $\left[r_{x}\left(P_{l}\right), r_{y}\left(P_{l}\right), r_{z}\left(P_{l}\right)\right]$ to have velocity $\left[v_{x}\left(P_{1}\right), v_{y}\left(P_{l}\right), v_{z}\left(P_{1}\right)\right]$ :

$$
\left\{\begin{array}{l}
v_{x}\left(P_{1}\right)=R_{1} \dot{\beta} \cos \beta \cos \varepsilon-R_{1} \dot{\varepsilon} \sin \beta \sin \varepsilon-h \dot{\beta} \sin \beta \\
v_{y}\left(P_{1}\right)=-R_{1} \dot{\beta} \sin \beta \cos \varepsilon-R_{1} \dot{\varepsilon} \cos \beta \sin \varepsilon-h \dot{\beta} \cos \beta \\
v_{z}\left(P_{1}\right)=-R_{1} \dot{\varepsilon} \cos \varepsilon
\end{array}\right.
$$

To calculate $K$, we had to get $v^{2}$ :

$$
v^{2}\left(P_{1}\right)=v_{x}^{2}\left(P_{1}\right)+v_{y}^{2}\left(P_{1}\right)+v_{z}^{2}\left(P_{1}\right)
$$

After reducing we had equation (10):

$v^{2}\left(P_{1}\right)=R_{1}^{2}\left(\beta^{2} \cos ^{2} \varepsilon+\varepsilon^{2}\right)+h^{2} \beta^{2}+2 R_{1} h \dot{\beta} \dot{\varepsilon} \sin \varepsilon$

\section{b) Position and velocity in the $2^{\text {nd }}$ subsystem:}

In Figure 2, the point $P_{2}$ was on the counterweight bar at a distance $R_{2}$ from the center of $B$ with coordinates in the Bxyz system of $\left[0,0,-R_{2}, 1\right]^{\mathrm{T}}$. The same calculation as (7), we got (11):

$$
\left\{\begin{array}{l}
r_{x}\left(P_{2}\right)=-R_{2} \sin \beta \sin \varepsilon+h \cos \beta \\
r_{y}\left(P_{2}\right)=-R_{2} \cos \beta \sin \varepsilon-h \sin \beta \\
r_{z}\left(P_{2}\right)=-R_{2} \cos \varepsilon
\end{array}\right.
$$

From (11), take the position derivative to get velocity, we got (12):

$$
v^{2}\left(P_{2}\right)=R_{2}^{2}\left(\beta^{2} \sin ^{2} \varepsilon+\varepsilon^{2}\right)+h^{2} \beta^{2}-2 R_{2} h \dot{\beta} \dot{\varepsilon} \cos \varepsilon
$$

c) Position and velocity in the $3^{\text {rd }}$ subsystem:

In Figure 2, the point $P_{3}$ was on the pivot pin at a distance $R_{3}$ from the center of $O$, whose coordinates in the Bxyz system were $\left[\left(R_{3}-h\right), 0,0,1\right]^{\mathrm{T}}$. Calculating according to (7), we got (13) and (14):

$$
\begin{aligned}
& \left\{\begin{array}{l}
r_{x}\left(P_{3}\right)=R_{3} \cos \beta \\
r_{y}\left(P_{3}\right)=R_{3} \sin \beta \\
r_{z}\left(P_{3}\right)=0
\end{array}\right. \\
& v^{2}\left(P_{3}\right)=R_{3}^{2} \beta^{2}
\end{aligned}
$$

\section{Kinetic and Potential Energy of Each Component}

Applying formulas (2) and (3) with the 3 components of TRMS as follows:

a) For members on the beam: the $1^{\text {st }}$ subsystem:

From (10) we have:

$$
\left\{\begin{aligned}
K_{1}= & \frac{1}{2}\left(\dot{\beta}^{2} \cos ^{2} \varepsilon+\dot{\varepsilon}^{2}\right) J_{1} \\
& +\frac{1}{2} h^{2} \dot{\varepsilon}^{2} m_{T_{1}}+h \dot{\beta} \dot{\varepsilon} m_{T_{1}} l_{T_{1}} \sin \varepsilon \\
V_{1}= & g m_{T_{1}} l_{T_{1}} \sin \varepsilon
\end{aligned}\right.
$$

Where:

$m_{T_{1}}$ - the total mass on the beam;

$l_{T_{1}}$ - the center of gravity of the beam;

$J_{1}$ - the inertia moment of the beam with the center of rotation $B$. 


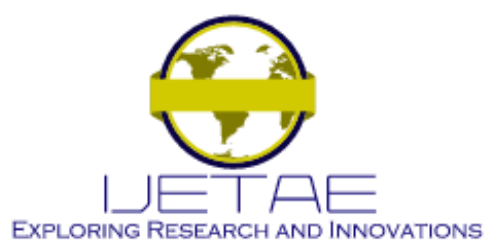

International Journal of Emerging Technology and Advanced Engineering

Website: www.ijetae.com (E-ISSN 2250-2459, Scopus Indexed, ISO 9001:2008 Certified Journal, Volume 11, Issue 08, August 2021)

$$
\begin{aligned}
m_{T_{1}}= & \int d m\left(R_{1}\right) \\
= & m_{t}+m_{t r}+m_{t s}+m_{m}+m_{m r}+m_{m s} \\
l_{T_{1}}= & \frac{\int R_{1} d m\left(R_{1}\right)}{\int d m\left(R_{1}\right)} \\
= & \frac{\left(m_{m} / 2+m_{m r}+m_{m s}\right) l_{m}}{m_{T_{1}}} \\
& +\frac{-\left(m_{t} / 2+m_{t r}+m_{t s}\right) l_{t}}{m_{T_{1}}} \\
J_{1}= & \int R_{1}^{2} d m\left(R_{1}\right) \\
= & \left(\frac{m_{t}}{3}+m_{t r}+m_{t s}\right) l_{t}^{2} \\
& +\left(\frac{m_{m}}{3}+m_{m r}+m_{m s}\right) l_{m}^{2} \\
& +\frac{1}{2} m_{m s} r_{m s}^{2}+m_{t s} r_{t s}^{2}
\end{aligned}
$$

Where:

$m_{t}, m_{t r}, m_{t s}, m_{m}, m_{m r}, m_{m s}$ - the mass of the beam on the tail rotor side, tail rotor, tail fender; the mass of the beam on the main rotor side, main rotor, and main fender, respectively;

$l_{m}, l_{t r}, r_{m s}, r_{t s}$ - the length and radius of the main beam, the tail beam, the main fender, and the tail fender, respectively.

b) For the counterweight: the $2^{\text {nd }}$ subsystem:

From (12) we got:

$$
\left\{\begin{aligned}
K_{2}= & \frac{1}{2}\left(\dot{\beta}^{2} \sin ^{2} \varepsilon+\dot{\varepsilon}^{2}\right) J_{2} \\
& +\frac{1}{2} h^{2} \dot{\beta}^{2} m_{T_{2}}-h \dot{\beta} \dot{\varepsilon} m_{T_{2}} l_{T_{2}} \cos \varepsilon \\
V_{2}= & -g m_{T_{2}} l_{T_{2}} \cos \varepsilon
\end{aligned}\right.
$$

Where:

$m_{T_{2}}$ - the total mass on the counterweight;

$l_{T_{2}}$ - the center of gravity of the counterweight bar including the counterweight;

$J_{2}$ - the inertia moment of the counterweight bar to the center of rotation $B$.

$$
\begin{gathered}
m_{T_{2}}=\int d m\left(R_{2}\right) \\
=m_{b}+m_{c b} \\
l_{T_{2}}=\frac{m_{b} l_{b} / 2+m_{c b} l_{c b}}{m_{T_{2}}} \\
J_{2}=\int R_{2}^{2} d m\left(R_{2}\right) \\
=\frac{1}{3} m_{b} l_{b}^{2}+m_{c b} l_{c b}^{2}+\frac{1}{4} m_{c b} r_{c b}^{2}+\frac{1}{12} m_{c b} L_{c b}^{2}
\end{gathered}
$$

With $l_{b}, l_{c b}, L_{c b}, m_{b}, m_{c b}$ were the counterweight bar length, counterweight to pivot distance, counterweight length, counterweight bar mass, and counterweight, respectively.

c) For the pivot pin: the $3^{\text {rd }}$ subsystem:

$$
\begin{aligned}
& \left\{\begin{array}{l}
K_{3}=\frac{1}{2} \dot{\beta}^{2} J_{3} \\
V_{3}=0
\end{array}\right. \\
& J_{3}=\int R_{3}^{2} d m\left(R_{3}\right) \\
& =\frac{m_{h}}{3} h^{2}
\end{aligned}
$$

\section{Energy Balance with Euler-Lagrange Equation}

From equation (1), take partial derivatives by azimuth and elevation angles as (18):

$$
\left\{\begin{array}{l}
\tau_{h}=\frac{d}{d t}\left(\frac{\partial L}{\partial \dot{\beta}}\right)^{T}-\left(\frac{\partial L}{\partial \beta}\right)^{T} \\
\tau_{v}=\frac{d}{d t}\left(\frac{\partial L}{\partial \dot{\varepsilon}}\right)^{T}-\left(\frac{\partial L}{\partial \varepsilon}\right)^{T}
\end{array}\right.
$$

Where:

- $\tau_{h}$ : The sum of the torque acting in the yaw motion (azimuth channel);

- $\tau_{v}$ : The sum of the moments acting in the pitch motion (elevation channel);

- L: The Lagrange energy function with 3 components taken according to formulas (15), (16a), (16b), (16c), and (17).

Ignoring the intermediate steps, we had the following results: 


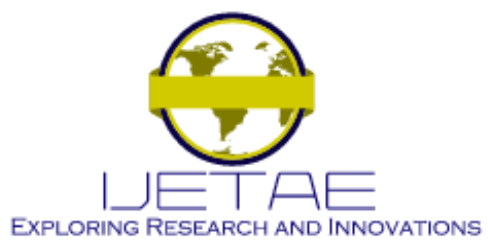

International Journal of Emerging Technology and Advanced Engineering

Website: www.ijetae.com (E-ISSN 2250-2459, Scopus Indexed, ISO 9001:2008 Certified Journal, Volume 11, Issue 08, August 2021)

$$
\begin{aligned}
\tau_{h}= & {\left[J_{1} \cos ^{2} \varepsilon+J_{2} \sin ^{2} \varepsilon+h^{2}\left(m_{T_{1}}+m_{T_{2}}\right)+J_{3}\right] \ddot{\beta} } \\
& +h\left(m_{T_{1}} l_{T_{1}} \sin \varepsilon-m_{T_{2}} l_{T_{2}} \cos \varepsilon\right) \ddot{\varepsilon} \\
& +h\left(m_{T_{1}} l_{T_{1}} \cos \varepsilon+m_{T_{2}} l_{T_{2}} \sin \varepsilon\right) \dot{\varepsilon}^{2} \\
& +2\left(J_{2}-J_{1}\right) \dot{\beta} \dot{\varepsilon} \sin \varepsilon \cos \varepsilon
\end{aligned}
$$

$$
\begin{aligned}
\tau_{v}= & \left(J_{1}+J_{2}\right) \ddot{\varepsilon} \\
& +h\left(m_{T_{1}} l_{T_{1}} \sin \varepsilon-m_{T_{2}} l_{T_{2}} \cos \varepsilon\right) \ddot{\beta} \\
& +g\left(m_{T_{1}} l_{T_{1}} \cos \varepsilon+m_{T_{2}} l_{T_{2}} \sin \varepsilon\right) \\
& +\dot{\beta}^{2}\left(J_{1}-J_{2}\right) \sin \varepsilon \cos \varepsilon
\end{aligned}
$$

Combining (19) and (20) and writing in vector form, we got the TRMS dynamics equation according to (21):

$$
\begin{aligned}
{\left[\begin{array}{c}
\tau_{h} \\
\tau_{v}
\end{array}\right]=} & {\left[\begin{array}{cc}
J_{1} \cos ^{2} \varepsilon+J_{2} \sin ^{2} \varepsilon+h^{2}\left(m_{T_{1}}+m_{T_{2}}\right)+J_{3} & h\left(m_{T_{1}} l_{T_{1}} \sin \varepsilon-m_{T_{2}} l_{T_{2}} \cos \varepsilon\right) \\
h\left(m_{T_{1}} l_{T_{1}} \sin \varepsilon-m_{T_{2}} l_{T_{2}} \cos \varepsilon\right) & J_{1}+J_{2}
\end{array}\right]\left[\begin{array}{l}
\ddot{\beta} \\
\ddot{\varepsilon}
\end{array}\right] } \\
& +\left[\begin{array}{c}
h\left(m_{T_{1}} l_{T_{1}} \cos \varepsilon+m_{T_{2}} l_{T_{2}} \sin \varepsilon\right) \dot{\varepsilon}^{2}+2\left(J_{2}-J_{1}\right) \dot{\beta} \dot{\varepsilon} \sin \varepsilon \cos \varepsilon \\
\dot{\beta}^{2}\left(J_{1}-J_{2}\right) \sin \varepsilon \cos \varepsilon+g\left(m_{T_{1}} l_{T_{1}} \cos \varepsilon+m_{T_{2}} l_{T_{2}} \sin \varepsilon\right)
\end{array}\right]
\end{aligned}
$$

The moment components applied to the TRMS in yaw motion include:

- The tail rotor thrust moment $M_{h}=l_{t} F_{h}\left(\omega_{h}\right) \cos \varepsilon$ (where $\omega_{h}$ - the angular velocity of the tail rotor and $F_{h}$ - the thrust of the tail fan wing);

- The frictional moment $M_{m s h}$;

- The drag torque produced by the $M_{c a b}$ signal cable

- The inter-channel moment $k_{m} \dot{\omega}_{v} \cos \varepsilon \quad\left(k_{m}\right.$ - the inter-channel factor and $\omega_{v}$ - the angular velocity of the main rotor).

The total torque acting on the TRMS in yaw motion:

$$
\tau_{h}=M_{h}-M_{m s h}-M_{c a b}+k_{m} \dot{\omega}_{v} \cos \varepsilon
$$

Similarly, the total torque acting on the TRMS during pitch motion:

$$
\tau_{v}=M_{v}-M_{m s v}+k_{t} \dot{\omega}_{h}+M_{g y r o}
$$

Where:

$M_{v}=l_{m} F_{v}\left(\omega_{v}\right)-$ the torque of the main rotor thrust ( $F_{v}$ - the main rotor thrust);

$M_{m s v}$ - the frictional moment in pitch motion;

$k_{t} \dot{\omega}_{h}$ - the inter-channel moment $\left(k_{t}-\right.$ the coefficient of inter-channel);
$M_{\text {gyro }}=k_{g} F_{v} \omega_{h} \cos \varepsilon$ - the component was caused by the gyroscope effect when the TRMS rotates in the horizontal plane with angular velocity $\omega_{h}=\dot{\beta}$.

From the mathematical model (21) along with all the parameters in (22), (23), we found that TRMS had the following basic characteristics:

- A nonlinear, uncertain system: a strong inter-channel due to the influence of the main and tail rotor movements; parameter uncertainty due to changes in the inertia moment inertia and coefficient of friction on joints; parameters could only be determined through identification.

- Being affected by noise, including system disturbance, measurement noise, noise caused by environmental conditions such as wind speed, temperature, humidity, etc.

Because of the above characteristics of the TRMS system, we need to have specialized control methods to be able to ensure the desired control quality. The block diagram of TRMS was shown in Figure 3.

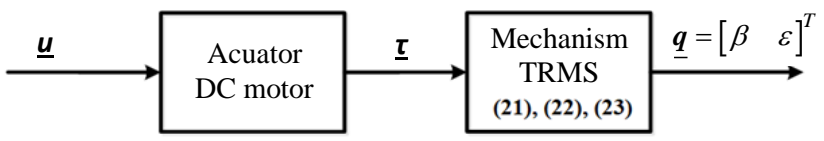

Figure 3. The block diagram of TRMS 


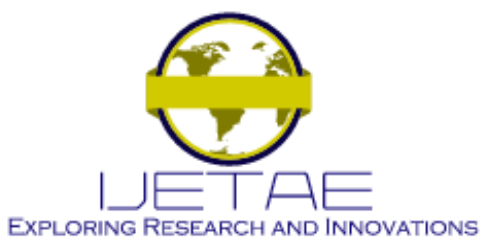

\section{International Journal of Emerging Technology and Advanced Engineering}

Website: www.ijetae.com (E-ISSN 2250-2459, Scopus Indexed, ISO 9001:2008 Certified Journal, Volume 11, Issue 08, August 2021)

In Figure 3, the main actuators are permanently excited DC motors, in which the usual control method is to change the field current or the armature voltage. With each different control algorithm approach, the control signal $\underline{\boldsymbol{u}}$ can be the angular velocity of the fans or the current and voltage supplied to the DC motors that rotate the fans.

\section{CONCLUSION}

The article proposed a new approach in building a dynamic model of the TRMS system based on the homogeneous coordinate transformation and the EulerLagrange equation on the view of separating the TRMS system into 3 subsystems. The model building process did not omit any mechanical details and a mechanical effect, so the result was a complete model with torque control input, serving the purpose of testing modern control algorithms.

In the next studies, the authors will evaluate the effectiveness of the model built with some algorithms of direct torque control with some sustainable adaptive controllers.

\section{Conflict of Interest}

The authors declare no conflict of interest.

\section{Acknowledgment}

The authors gratefully acknowledge the constructive and insightful comments of reviewers to further improve the quality of this paper. This work was supported in part by a grant from CAPITI.

\section{REFERENCES}

[1] Feedback Instruments Ltd. 1997. Twin Rotor MIMO System Technique Manual 33-007-4M5. England.

[2] Feedback Instruments Ltd ES, TN6 2QR. 2006. Twin Rotor MIMO System Control Experiments 33-949S. UK.

[3] Rahideh A., Shaheed MH. 2007. Mathematical dynamic modeling of twin-rotor multiple inputs - multiple outputs system. Proceedings of the Institution of Mechanical Engineers, Part I - Journal of Systems and Control Engineering, 221 (I1), pp. 89-101.

[4] Rahideh A. 2009. Model Identification and Robust Nonlinear Model Predictive of a Twin Rotor MIMO System. Ph.D. Thesis. School of Engineering and Materials Science Queen Mary. University of London.

[5] Petr Chalupa, Jan Přikryl, Jakub Novák. 2014. Modeling of Twin Rotor MIMO System, 25th DAAAM International Symposium on Intelligent Manufacturing and Automation. DAAAM, pp. 249-258.

[6] Sarvat Mushtaq Ahmad B. 2001. Modeling and Control of a Twin Rotor MIMO System. Ph.D. Thesis. The University of Sheffield. UK.

[7] Asutosh Satapathy, Rashmi Ranjan Nayak. 2010. Modeling of Twin Rotor MIMO System (TRMS). Ph.D. Thesis. National Institute of Technology Rourkela. India.

[8] Azamat Tastemirov, Andrea Lecchini-Visintini, Rafael M. MoralesViviescas. 2017. Complete dynamic model of the Twin Rotor MIMO System (TRMS) with experimental validation. Control Engineering Practice. Vol. 66. pp 89-98.

[9] T. Dang Huu, I. B. Ismail. 2016. Modelling of Twin Rotor MIMO system. 2016 2nd IEEE International Symposium on Robotics and Manufacturing Automation (ROMA). pp. 1-6, doi: 10.1109/ROMA.2016.7847803. 